\title{
A collagen thin film-based bioimpedance sensor for cell proliferation rate assessment
}

\author{
Masoomeh Ashoorirad ${ }^{1}$, Rasool Baghbani ${ }^{2}$, Ali Fallah², Najmeh Jooyan ${ }^{2}$, and Maryam \\ Ghaffari $^{2}$ \\ ${ }^{1}$ Hamedan University of Technology \\ ${ }^{2}$ Affiliation not available
}

May 14, 2021

\begin{abstract}
In this paper, a bioimpedance sensor used to measure the impedance behavior of the biological cells cultured on a scaffold of collagen thin films. The collagen thin films with different concentrations were created on a 1-hexadecanethiol modified surface of the interdigitated electrodes(IDEs). The mesenchymal stem cells(MSCs) and human umbilical vein endothelial cells(HUVECs) were cultured on the collagen thin films. The interaction and the proliferation rate of these cells were investigated by the microscopic, and bioimpedance approaches. Results showed that the MSCs, and HUVECs excellently attached to the collagen thin films. Impedance measurements of the cells cultured on the collagen thin film were performed by a Hioki IM3570 impedance analyzer at the frequency band of $10 \mathrm{kHz}$ to $1 \mathrm{MHz}$ at $10 \mathrm{mV}$. Bioimpedance measurements showed that the proliferation rate of both MSCs, and HUVECs increased by decreasing the collagen thin films concentration. After $48 \mathrm{~h}$, the differential impedances $(\Delta \mathrm{Z})$ of the MSCs cultured on the collagen thin layers with concentrations of $250 \mu \mathrm{g} / \mathrm{mL}, 500 \mu \mathrm{g} / \mathrm{mL}, \mathrm{and} 1000 \mu \mathrm{g} / \mathrm{mL}$ measured $3.09 \Omega[?] \mathrm{D}[?] 48.03 \Omega, 2.62 \Omega[?] \mathrm{D}[?] 45.79 \Omega$, and $1.9 \Omega[?] \mathrm{D}[?] 25.22 \Omega$, respectively. Also, $\Delta \mathrm{Z}$ of the HUVECs obtained $27.3 \Omega[?] \mathrm{D}[?] 39.2 \Omega, 8.25 \Omega[?] \mathrm{D}[?] 18.43 \Omega$, and $0.96 \Omega[?] \mathrm{D}[?] 9.6 \Omega$ for the corresponding frequency band and collagen thin films concentrations.
\end{abstract}

\section{A collagen thin film-based bioimpedance sensor for cell proliferation rate assessment}

M. Ashoorirad ${ }^{1, *}$, R. Baghbani ${ }^{1}$, A. Fallah ${ }^{2}$, N. Jooyan ${ }^{3}$, M. Ghaffari ${ }^{2}$

${ }^{1}$ Biomedical Engineering Department, Hamedan University of Technology, Hamedan, Iran.

${ }^{2}$ Biomedical Engineering Department, Amirkabir University of Technology (Tehran Polytechnic), Tehran, Iran.

${ }^{3}$ Department of Medical Physics and Biomedical Engineering, School of Medicine, Shiraz University of Medical Sciences, Shiraz, Iran.

\begin{abstract}
In this paper, a bioimpedance sensor used to measure the impedance behavior of the biological cells cultured on a scaffold of collagen thin films. The collagen thin films with different concentrations were created on a 1hexadecanethiol modified surface of the interdigitated electrodes(IDEs). The mesenchymal stem cells(MSCs) and human umbilical vein endothelial cells(HUVECs) were cultured on the collagen thin films. The interaction and the proliferation rate of these cells were investigated by the microscopic, and bioimpedance approaches. Results showed that the MSCs, and HUVECs excellently attached to the collagen thin films. Impedance measurements of the cells cultured on the collagen thin film were performed by a Hioki IM3570 impedance analyzer at the frequency band of $10 \mathrm{kHz}$ to $1 \mathrm{MHz}$ at $10 \mathrm{mV}$. Bioimpedance measurements
\end{abstract}


showed that the proliferation rate of both MSCs, and HUVECs increased by decreasing the collagen thin films concentration. After $48 \mathrm{~h}$, the differential impedances () of the MSCs cultured on the collagen thin layers with concentrations of ,, and measured, , and , respectively. Also, of the HUVECs obtained, , and for the corresponding frequency band and collagen thin films concentrations.

Keywords: Collagen Thin film; Alkanethiolate-treated electrodes; Bioimpedance spectroscopy; Biological cells.

\section{Introduction}

The main challenge encountering the biotechnology is the capability of quantitative evaluation of biomarker expression in living cells. Toxicology and drug screening rely on cell-based approaches, hoping that quantitative cell respond will demonstrate a more trusty foreteller of clinical results than molecular-scale approaches ${ }^{[1]}$. The development of modified matrices and quantitative techniques help obtain more reliable, reproducible, and meaningful cell-based measurements. Thin films of extracellular matrix (ECM) proteins have been used as helpful apparatuses in various biological examinations ${ }^{[2]-[9]}$, which give controlled surfaces to cell-based tests. A controlled surface is vital for the examination of cell proliferation, migration, and differentiation ${ }^{[7]}$.

Collagen is the primary fibrous protein in the ECM. In type I collagen molecule, there are three amino acid chains that form rod-shaped triple helices. The triple helices are assembled and form fibrils; then bundles and fibers are formed by lateral aligning of the fibrils ${ }^{[10], ~[11]}$. Thin films of collagen are beneficial to coordinate different biological issues, for example, cell morphology ${ }^{[4]},[6],[12]$, the impact of surface attributes on intracellular flagging ${ }^{[4]}$, and the collaboration between the surface and integrin receptors ${ }^{[8]}$. These films elicit the same cell conduct as thick collagen gels, but they are generally more reproducible and simpler to depict ${ }^{[3]}$. The collagen thin films are more straightforward than thick collagen gels so that singular fibrils can be portrayed by optical microscopy, permitting more thorough investigations of the connection among cells and fibrils ${ }^{[7]}$. Besides, a disadvantage of thick collagen gels is that they are challenging to plan methodically from one trial to another ${ }^{[3]}$. The collagen gels can easily detach from their supporting surfaces, and visible variety in the gel can be seen by the eye. Also, quantitative examination of cell attributes on these matrices can be convoluted on account of light scattering from the fibrillar gel and catching of fluorescent reagents ${ }^{[3]}$.

Bioimpedance points out the objection of the biological specimens like bacteria, biological cells, and living tissues to the electric current stream. Bioimpedance measurements are progressively used in biomedical utilizations and cell investigations ${ }^{[13]-[17]}$. Electrical cell-substrate impedance sensing (ECIS) is the phrase given to a specific kind of bioimpedance measurement explicitly pertinent to measuring the electrical impedance of adherent living cells on planar microelectrodes ${ }^{[18]}$. ECIS has been utilized to show various properties of cells, for example, attachment and spreading ${ }^{[19]-[23]}$, motility ${ }^{[24],[25]}$, and growth and proliferation ${ }^{[26],[27]}$. Bioimpedance analyses have been used as a reliable means to distinguish healthy and cancerous cells and to determine metastatic attributes ${ }^{[28]-[37]}$. Impedance measurements of adherent cells can discover variations in cell morphology on the order of nanometers ${ }^{[38]}$, providing sensitivity more significant than that got by visual inspections. Generally, impedance measurements can discover a small variation in cell capacitance and resistance attached to the measuring microelectrodes.

In our previous work, the collagen thin films were created on the electrode surface modified by oxygen plasma; and the impact of the deionized water on the measured impedance of the collagen thin films was examined [39]. Results showed that the collagen thin films impedance in the presence of deionized water decreased by increasing the collagen thin films concentration. Due to the dependence of the collagen thin films impedance on the collagen concentration, in the cellular experiments, the proliferation rate of the cells has been investigated using the differential impedances () during the time.

In this paper, we portray the preparation and characterization of collagen thin films on alkanethiol-treated gold interdigitated electrodes (IDEs) and the evaluation of their biomimetic nature by microscopic approaches such as field-effect scanning electron microscopy (FESEM). Then, the proliferation rate of mesenchymal stem cells derived from rabbit adipose tissue (MSCs), malignant breast cancer cells (MDA-MB-231), and 
human umbilical vein endothelial cells (HUVEC) on the collagen thin films with different concentrations was investigated by bioimpedance measurements. The use of fibrillar collagen thin films offers excellent cellular collaboration with the collagen scaffold and reproducibility of the scaffold preparation.

\section{Materials and methods}

\section{Biosensor construction}

Microscope glass slides, as a substrate for electrodes, were cleaned through the standard RCA \#1 method (a mixed solution of $\mathrm{NH}_{4} \mathrm{OH}: \mathrm{H}_{2} \mathrm{O}_{2}: \mathrm{H}_{2} \mathrm{O}$ in volume ratio of 1:1:5, respectively). Subsequently, a thin layer of Ti $(50 \mathrm{~nm})$ was deposited on the glass substrate by sputtering to improve the gold adhesion to the substrate. A $200 \mathrm{~nm}$ thin layer of gold was deposited on Ti by a sputtering system (Veeco Co.). The gold was planned using soft lithography and the design of the IDEs with $100 \mu \mathrm{m}$ width and a distance of $100 \mu \mathrm{m}$ between every two branches that transferred on the glass substrate.

\section{Collagen solutions preparation}

Dry Type 1 Collagen, gotten from calf skin, was bought from the Zist Farayand Tajhiz Sahand (Tabriz). A sum of $36 \mathrm{mg}$ of collagen was dissolved in $12 \mathrm{ml}$ of $0.4 \mathrm{M}$ acetic acid (Sigma Aldrich, CAS No: 64-19-7) using a magnetic stirrer at 4 (refrigerator); and a stock solution of $3 \mathrm{mg} / \mathrm{ml}$ concentration was prepared. Before dissolving, dry collagen was exposed to ultraviolet radiation for 5-10 minutes to be sterilized. To prepare the neutralized collagen solution, the cold stock solution was blended with the cold concentrated (10X) PBS, and cold $0.5 \mathrm{M} \mathrm{NaOH}$ (Sigma Aldrich, CAS No. 1310-73-2) in a 8:1:1 ratio to attain physiologically PH and ionic strength conditions under which collagen monomer effectively polymerizes into fibrils at 37 . The obtained neutralized solution was diluted with (1X) PBS, to obtain the desired concentration of collagen solution. The collagen solution was neutralized on ice to keep the collagen from polymerization during the neutralization process.

\section{Preparation of alkanethiol-coated interdigitated electrodes}

Chambers of Plexiglas were created and attached to the substrates in a way that embedded the fabricated electrodes. The amount of $600 \mu \mathrm{L}$ of $2 \mathrm{mM}$ 1-hexadecanethiol (Sigma Aldrich, CAS No: 2917-26-2 ) solution in absolute ethanol (Sigma Aldrich, CAS No. 64-17-5) was poured in the chambers and remained overnight under the biological flow hood. After that, the electrodes were washed with ethanol and dried under biological flow hood before incubation with collagen solution. Alkanethiol-coated samples could be held under ethanol at least for seven days without any damage in efficiency ${ }^{[3]}$. 1-hexadecanethiol is a chemical material with formula $\mathrm{CH}_{3}\left(\mathrm{CH}_{2}\right)_{15} \mathrm{SH}$; its head group $(\mathrm{SH})$ chemically connected to the gold electrodes, and its hydrophobic tail group $\left(\mathrm{CH}_{3}\right)$ creates a chemical bond with the collagen molecules.

\section{Preparation of type 1 collagen thin films}

The amount of $400 \mu \mathrm{L}$ of cold neutralized collagen solution was poured in each of the alkanethiol-treated IDEs and then were incubated overnight at 37 in a $5 \% \mathrm{CO}_{2}$ incubator to permit collagen polymerization. Collagen thin films were constructed by permitting collagen molecules to absorb to a hydrophobic alkanethiol surface. The collagen-coated electrodes were rinsed several times with (1X) PBS, and then with sterile deionized water to remove all loosely adhered collagen molecules or fibrils from the surface. Subsequently, the samples were briefly dried under a steam of air under biological flow hood and then placed into a (1X) PBS solution at 4 ready for use with cells.

For characterization of the collagen thin films, the samples were pictured using a field-effect scanning microscopy (FESEM). Images were obtained from various regions on any sample to make certain the homogeneity of surface specifications. The FESEM images of the created collagen thin films of various concentrations on the 1-hexadecanethiol-treated IDEs is shown in Fig. 1 

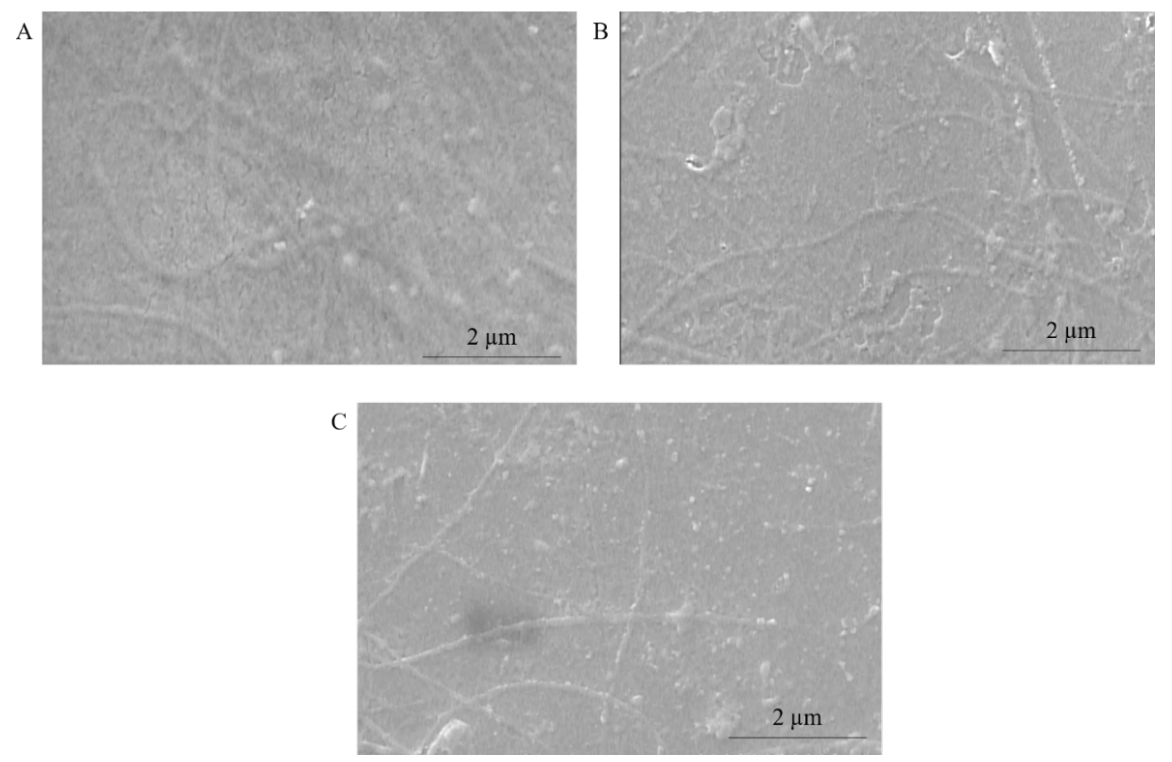

Fig. 1: FESEM images of type 1 collagen fibrils with different concentrations created on 1-hexadecanethioltreated IDEs; (A) $1000 \mu \mathrm{g} / \mathrm{mL}$, (B) $500 \mu \mathrm{g} / \mathrm{mL}$, (C) $250 \mu \mathrm{g} / \mathrm{mL}$.

\section{Cell culture on collagen thin films}

The MSCs, HUVEC, and MDA-MB-231 cell lines were bought from the National Cell Bank of Iran (Pasteur Institute, Iran). They were cultured in Dulbecco's modified Eagle's medium (DMEM) (Mediatech, Herndon, VA) supplemented with 10\% fetal bovine serum (FBS; Gibco Invitrogen, Carlsbad, CA) and 1\% penicillin/streptomycin (Gibco). Before seeding cells on the alkanethiol-treated electrodes containing collagen thin films, the thin films were covered in DMEM medium with 5\% FBS at least for $3 \mathrm{~h}$ three times. This step was required to normalize the collagen thin film concerning likely absorption of serum proteins ${ }^{[3]}$. Cells were detached from culture plates by trypsinization, rinsed with DMEM culture medium having $5 \%$ FBS, and then centrifuged for 3 minutes at $1000 \mathrm{rpm}$. Then, the suspended cells in DMEM medium with $5 \%$ FBS covered on the thin films of collagen at a $2 \times 10^{4}$ cells $/ \mathrm{cm}^{2}$ density and held at $37{ }^{\circ} \mathrm{C}$ for $48 \mathrm{~h}$. The decreased concentrations of serum maximizes the amount of cell signaling owning to the extracellular matrix ${ }^{[3]}$. Fig. 2 (C-D) shows the microscopic images of MSCs and HUVEC cells on collagen thin films with various concentrations.

\section{Impedance measurements}

Impedance measurements of cells on the collagen thin film were performed by a Hioki IM3570 impedance analyzer across a frequency band of $10 \mathrm{kHz}$ to $1 \mathrm{MHz}$ at $10 \mathrm{mV}$. Fig. 2 shows the impedance measurement setup. 


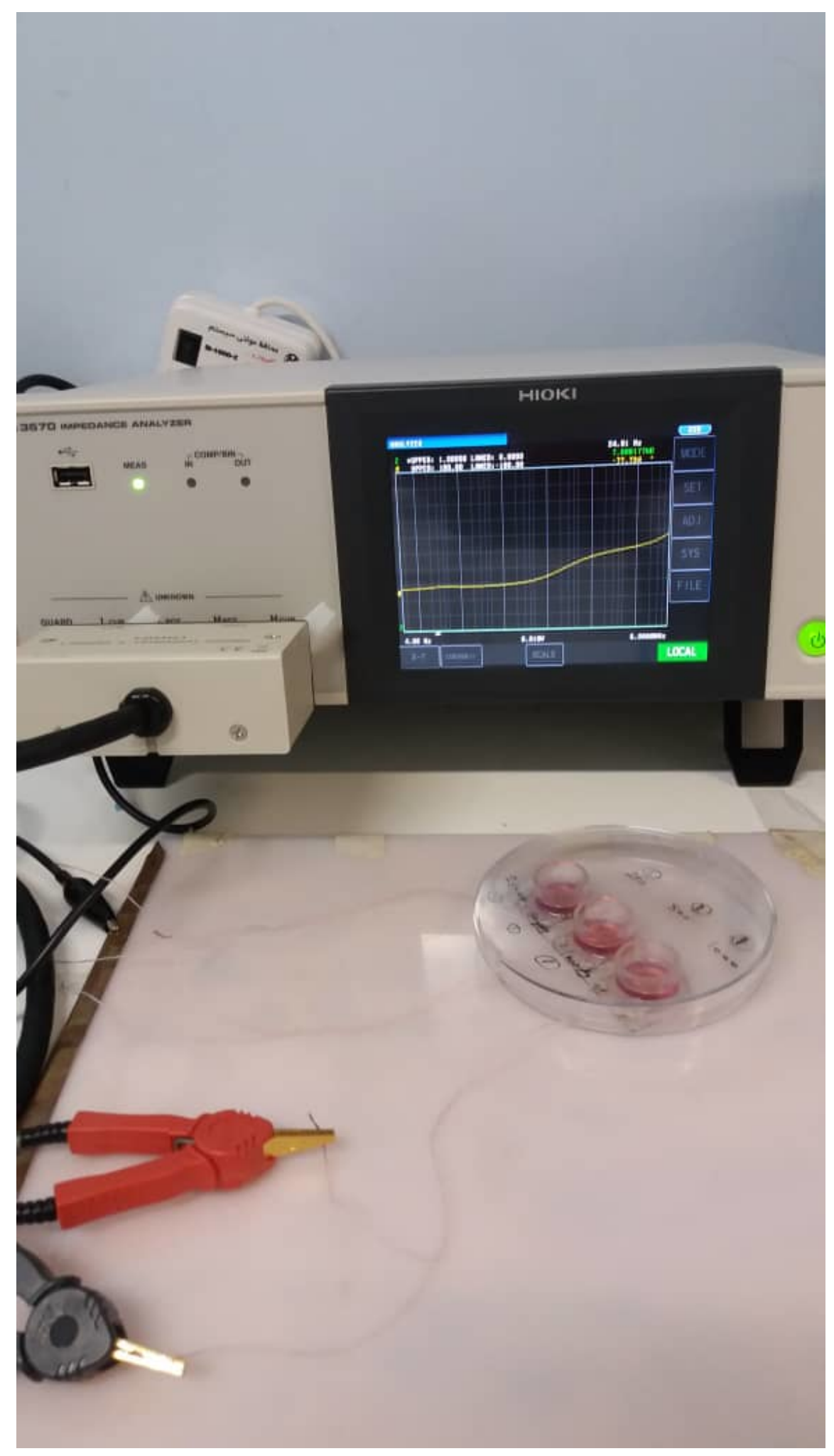

Fig. 2: (A) Measurement setup for measuring cellular impedance, (B) measuring interdigitated electrodes 
(IDEs). Optical microscopic images of (C) the MSCs on collagen thin films of $250 \mu \mathrm{g} / \mathrm{mL}$ concentration and (D) the HUVECs on collagen thin films of $500 \mu \mathrm{g} / \mathrm{mL}$ concentration.

\section{Statistical Analysis}

Obtained results were analyzed utilizing SPSS 22 software. Data were indicated as means \pm standard error of the mean. All data were expressed as the mean of three repeats. At each repeat of every concentration, at least three samples were measured. The means were compared by student's T-test. Discrepancies were evaluated to be statistically significant at $\mathrm{p}<0.05$.

\section{Results and discussion}

\section{Effect of concentration of collagen thin films on the viability of the MSCs cells}

The MSCs were cultured in chambers on top of the IDEs modified with a self-assembled monolayer (SAM) of 1-hexadecanethiol. After $48 \mathrm{~h}$, cell viability was detected via the dye exclusion method using trypan blue staining. The obtained results are shown in Fig. 3 (A) for different concentrations of collagen thin films. As shown in the diagrams, there is no substantial difference among the viability of MSCs cells cultured on the collagen thin films with various concentrations.

\section{Hosted file}

image22.emf available at https://authorea.com/users/413813/articles/522008-a-collagen-thinfilm-based-bioimpedance-sensor-for-cell-proliferation-rate-assessment

Fig. 3: (A) The obtained viability of the MSCs cultured on the collagen thin films with various concentrations after $48 \mathrm{~h}$; (B) Results of counting the number of MSCs on the collagen thin films with various concentrations after $48 \mathrm{~h}$. Symbol * represents statistically significant between groups determined by ANOVA $(\mathrm{p}<0.05)$.

Bioimpedance assessment of the collagen thin films concentration on the MSCs proliferation rate

\subsubsection{Bioimpedance magnitude of the MSCs cells cultured on the collagen thin film}

Fig. 4 (A-C) shows the magnitude diagram of the measured impedances of the MSCs seeded on the collagen thin films with various concentrations during the time. As shown in the diagram, the magnitude of the measured differential bioimpedance () at different concentrations of collagen thin films has increased over time. This increase in impedance can be due to cell attachment and proliferation over time. The diagram of the of the MSCs cultured on different concentrations of collagen thin films after $48 \mathrm{~h}$ is shown in Fig. 5 (A). Results showed that after $48 \mathrm{~h}$, when the doubling time of the MSCs has elapsed, the amount of increase in impedance at lower concentrations is higher than that of higher concentrations, which may indicate more cell growth on the collagen thin films with lower concentration. Previous studies have investigated the relationship between the collagen thin films concentration and cell proliferation and morphology [2]-[6], [8], [40] . The cells that interact with fibrils formed from higher concentrations of collagen solution do not spread much on the surface and show a weaker cytoskeleton than the cells that interact with fibrils formed from lower concentrations of the collagen thin films. The cells on the surfaces prepared from low collagen concentrations have a higher rate of proliferation and show variations in gene expression compared to the cells on collagen with similar concentrations to the physiological condition ${ }^{[41]}$. The important point of this research is that we have concluded this result with a quantitative impedance criterion. The same results obtained for HUVECs cells under similar conditions, which will represent in the next section.

\section{Hosted file}

image26.emf available at https://authorea.com/users/413813/articles/522008-a-collagen-thinfilm-based-bioimpedance-sensor-for-cell-proliferation-rate-assessment

Fig. 4: The differential bioimpedance () of the MSCs and HUVECs cultured on collagen thin films with concentrations of $250 \mu \mathrm{g} / \mathrm{mL}, 500 \mu \mathrm{g} / \mathrm{mL}, 1000 \mu \mathrm{g} / \mathrm{mL}$ over time. 


\section{Hosted file}

image32.emf available at https://authorea.com/users/413813/articles/522008-a-collagen-thinfilm-based-bioimpedance-sensor-for-cell-proliferation-rate-assessment

The number of cells counted using neobar lam (hemocytometer) also showed that the lower the concentration of collagen thin films, the higher the number of cells after $48 \mathrm{~h}$. Results are shown in Fig. 3 (B).

Fig. 5: The differential bioimpedance of the cells (A) the MSCs, and (B) the HUVECs cultured on the collagen thin films with different concentrations, $48 \mathrm{~h}$ after their seeding.3.2.2 Bioimpedance phase of the MSCs cultured on the collagen thin film

The differential phase $(\varphi)$ diagrams of the measured bioimpedance of the cultured MSCs on the collagen thin films with different concentrations over time are shown in Fig. 6 (A-C). As shown in these graphs, for all concentrations of collagen thin films, the impedance phase has become more negative over time, indicating the attachment and growth of cells over time. The lower the concentration of the collagen thin films, the greater the amount of this negativity, showing the higher number of the cells inside the wells. In other words, from the measured impedance phase, it can be seen that over time, the cells have more attachment and proliferation rate on the collagen thin films with lower concentration with respect to higher concentrations, which makes the phase of bioimpedance more negative.

\section{Hosted file}

image40.emf available at https://authorea.com/users/413813/articles/522008-a-collagen-thinfilm-based-bioimpedance-sensor-for-cell-proliferation-rate-assessment

Fig. 6: Changes of the impedance phase of MSCs and HUVECs cultured on collagen thin films with concentrations of $250 \mu \mathrm{g} / \mathrm{mL}, 500 \mu \mathrm{g} / \mathrm{mL}, 1000 \mu \mathrm{g} / \mathrm{mL}$ over time.

\section{Effect of the collagen thin films on the MDA-MB-231 cells}

In the second step of the research, tests were accomplished on the MDA-MB-231 to explore the effect of the collagen thin film concentration on the growth and proliferation of the cancer cells. This experiment was performed twice for four concentrations and five samples from each concentration. These cells did not attach to the collagen thin films in any of the samples and died. In general, cancer cells produce collagenase. Collagenase is an enzyme that dissolves and destroys collagen. In particular, MDA-MB-231 cells, which are aggressive and malignant, show this behavior more strongly. Three mammalian collagenases (MMP-1, MMP-8, MMP-13) [42] appertain to the group of matrix metalloproteinases and can cleave and destroy the extracellular collagen matrix ${ }^{[42]}$. In addition to the fibrillar collagens, collagenases can cleave and destroy some other extracellular matrices and non-matrix proteins, including growth factors ${ }^{[42]}$. MMPs are commonly thought of as tumor growth enzymes because they destroy extracellular matrix components and thus increase the invasive power of the cancerous cells ${ }^{[3]}$. It seems that due to the fact that these collagenases have been observed in breast cancerous cells ${ }^{[4]}$, they can destroy the thin layers of the collagen. By destroying these thin layers, which are considered as a bed for the cell attachment, the cells do not found an appropriate substrate to attach and die.

\section{Bioimpedance assessment of the collagen thin films concentration on the HUVECs proliferation rate}

In the third step of the research, collagen thin films were created on the surface of the alkanethiol-treated IDEs and the role of the concentration of the collagen thin films on the growth and proliferation of the HUVECs was quantitatively investigated using impedance measurements. Like the MSCs, viability assessment of HUVECs showed no remarkable discrepancy among the viability of HUVECs cultured on the collagen thin films with different concentrations. The HUVECs cultured on the collagen thin films with various concentrations had a viability of over $99 \%$. As well, obtained impedance results were analogous with the results of the MSCs.

\subsubsection{Bioimpedance magnitude of the HUVECs cultured on the collagen thin films}


The impedance measurement results of the HUVECs were similar to that of the MSCs. Fig. 4 (D-F) shows the magnitude diagram of the measured impedances of the HUVECs cultured on the collagen thin films with different concentrations during the time. As shown in the figure, the magnitude of the measured bioimpedance at different concentrations of collagen thin films has increased over time. This increase in the impedance in the first hours results from the cell attachment. After $48 \mathrm{~h}$, the increased impedance magnitude is due to the cell proliferation since the cell numbers have been increased because cells doubling time has elapsed. The diagram of the measured differential bioimpedance for different concentrations after $48 \mathrm{~h}$ is also shown in Fig. 5 (B). We can see in this figure that after $48 \mathrm{~h}$, when the doubling time (about $36 \mathrm{~h}$ ) of the HUVECs has elapsed, the amount of increase in impedance at lower concentrations is higher than that of the higher concentrations, which may indicate more cell growth on the collagen thin films with lower concentration.

\subsubsection{Bioimpedance phase of the HUVECs on the collagen thin films}

Fig. 6 (D-F) shows the phase diagrams of the measured bioimpedance of the cultured HUVECs on the collagen thin films with different concentrations during the time. The obtained results were consistent with the results of the MSCs. As shown in the figure, for all concentrations of the collagen thin films, the impedance phase has become more negative over time, indicating the attachment and growth of cells over time. The lower the concentration of the collagen thin films, the greater the amount of this negativity, indicating the greater number of the cells inside the wells. Further negation of the impedance phase over time indicates that the number of cells has increased. Also, similar to the MSCs, the cells cultured on the collagen thin films with lower concentration, has more negative differential phase, which indicates that the proliferation rate of the cells on the low concentration collagen thin films is higher respect to the cells cultured on the collagen thin films with higher concentrations.

\section{Conclusion}

In this paper, a bioimpedance sensor was used to measure the impedance behavior of the biological cells cultured on a scaffold of collagen thin films. The collagen thin films were formed on the 1-hexadecanethiol modified surface of the interdigitated electrodes. Collagen thin films are beneficial to direct a variety of biological issues such as cell morphology, cell proliferation, cell migration, and the effect of surface characteristics on the cellular behaviors. Three different cell lines, the MSCs, the MDA-MB-231, and HUVECs were seeded on the collagen thin films with various concentrations. The interaction and the proliferation rate of these cells were investigated by the microscopic and impedance approaches. Results showed that the MDA-MB-231 cells did not attach to the collagen thin films and died. In general, cancerous cells produce collagenase, which is an enzyme that dissolves and destroys collagen. It seems since these collagenases exist in breast cancerous cells, they can destroy the collagen thin films and consequently, the appropriate bed for cell attachment is destroyed. By destroying these collagen thin films, the cells do not find a proper substrate to adhere and die.

The MSCs, and HUVECs excellently adhered to the collagen thin films and were investigated by microscopic and impedance methods. Impedance magnitude and phase illustrated that the proliferation rate of both MSCs, and HUVECs increased by reducing the collagen thin films concentrations.

\section{Conflict of interest statement}

We, Masoomeh Ashoorirad, Rasool Baghbani, Ali Fallah, Najmeh jooyan, and Maryam Ghaffari guarantee that there is no contrast of interest.

\section{Acknowledgment}

This research was supported by Iran National Science Foundation (INSF) through the financial agreement number 97015120. The authors would like to thank Dr. Reza Faraji_Dana for his support. This research was performed in his Bioelectromagnetic laboratory at Tehran University.

\section{References}


[1] H. D. Taylor, J. R. Haskins, and K. A. Giuliano, High content screening: A powerful approach to systems cell biology and drug discovery totowa . 2007.

[2] J. T. Elliott, M. Halter, A. L. Plant, J. T. Woodward, K. J. Langenbach, and A. Tona, "Evaluating the performance of fibrillar collagen films formed at polystyrene surfaces as cell culture substrates," Biointerphases, vol. 3, no. 2, pp. 19-28, 2008.

[3] J. T. Elliott, A. Tona, J. T. Woodward, P. L. Jones, and A. L. Plant, "Thin films of collagen affect smooth muscle cell morphology," Langmuir, vol. 19, no. 5, pp. 1506-1514, 2003.

[4] J. T. Elliott, J. T. Woodward, K. J. Langenbach, A. Tona, P. L. Jones, and A. L. Plant, "Vascular smooth muscle cell response on thin films of collagen," Matrix Biol. , vol. 24, no. 7, pp. 489-502, 2005.

[5] J. T. Elliott, J. T. Woodward, A. Umarji, Y. Mei, and A. Tona, "The effect of surface chemistry on the formation of thin films of native fibrillar collagen," Biomaterials, vol. 28, no. 4, pp. 576-585, 2007.

[6] K. J. Langenbach, J. T. Elliott, A. Tona, D. McDaniel, and A. L. Plant, "Thin films of Type 1 collagen for cell by cell analysis of morphology and tenascin-C promoter activity," BMC Biotechnol. , vol. 6, no. 1, p. $14,2006$.

[7] F. Amyot et al. , "Thin films of oriented collagen fibrils for cell motility studies," J. Biomed. Mater. Res. Part B Appl. Biomater. An Off. J. Soc. Biomater. Japanese Soc. Biomater. Aust. Soc. Biomater. Korean Soc. Biomater. , vol. 86, no. 2, pp. 438-443, 2008.

[8] D. P. McDaniel et al. , "The stiffness of collagen fibrils influences vascular smooth muscle cell phenotype," Biophys. J. , vol. 92, no. 5, pp. 1759-1769, 2007.

[9] N. L. Nerurkar, D. M. Elliott, and R. L. Mauck, "Mechanics of oriented electrospun nanofibrous scaffolds for annulus fibrosus tissue engineering," J. Orthop. Res. , vol. 25, no. 8, pp. 1018-1028, 2007.

[10] A. Stylianou and D. Yova, "Surface nanoscale imaging of collagen thin films by Atomic Force Microscopy," Mater. Sci. Eng. C, vol. 33, no. 5, pp. 2947-2957, 2013.

[11] A. Stylianou, D. Yova, and E. Alexandratou, "Investigation of the influence of UV irradiation on collagen thin films by AFM imaging," Mater. Sci. Eng. C, vol. 45, pp. 455-468, 2014.

[12] C. S. Chen, E. Ostuni, G. M. Whitesides, and D. E. Ingber, "Using Self-Assembled Monolayers to Pattern ECM Proteins and Cells on Subtrates," in Extracellular Matrix Protocols, Springer, 2000, pp. 209-219.

[13] G. Linz, S. Djeljadini, L. Steinbeck, G. Kose, F. Kiessling, and M. Wessling, "Cell barrier characterization in transwell inserts by electrical impedance spectroscopy," Biosens. Bioelectron. , p. 112345, 2020.

[14] K. Heileman, J. Daoud, and M. Tabrizian, "Dielectric spectroscopy as a viable biosensing tool for cell and tissue characterization and analysis," Biosens. Bioelectron. , vol. 49, pp. 348-359, 2013.

[15] F. M. Spiga et al. , "Hybridization chain reaction performed on a metal surface as a means of signal amplification in SPR and electrochemical biosensors," Biosens. Bioelectron., vol. 54, pp. 102-108, 2014.

[16] I. O. K'Owino and O. A. Sadik, "Impedance spectroscopy: a powerful tool for rapid biomolecular screening and cell culture monitoring," Electroanal. An Int. J. Devoted to Fundam. Pract. Asp. Electroanal. , vol. 17, no. 23, pp. 2101-2113, 2005.

[17] T. Sun and H. Morgan, "Single-cell microfluidic impedance cytometry: a review," Microfluid. Nanofluidics, vol. 8, no. 4, pp. 423-443, 2010.

[18] I. Giaever and C. R. Keese, "A morphological biosensor for mammalian cells.," Nature, vol. 366, no. 6455, pp. 591-592, 1993.

[19] T. Anh-Nguyen, B. Tiberius, U. Pliquett, and G. A. Urban, "An impedance biosensor for monitoring cancer cell attachment, spreading and drug-induced apoptosis," Sensors Actuators A Phys. , vol. 241, pp. 
231-237, 2016.

[20] J. Wegener, C. R. Keese, and I. Giaever, "Electric cell-substrate impedance sensing (ECIS) as a noninvasive means to monitor the kinetics of cell spreading to artificial surfaces," Exp. Cell Res. , vol. 259, no. 1, pp. 158-166, 2000.

[21] P. Mitra, C. R. Keese, and I. Giaever, "Electric measurements can be used to monitor the attachment and spreading of cells in tissue culture.," Biotechniques, vol. 11, no. 4, pp. 504-510, 1991.

[22] F. Asphahani et al. , "Influence of cell adhesion and spreading on impedance characteristics of cell-based sensors," Biosens. Bioelectron. , vol. 23, no. 8, pp. 1307-1313, 2008.

[23] H. Abiri et al. , "Monitoring the spreading stage of lung cells by silicon nanowire electrical cell impedance sensor for cancer detection purposes," Biosens. Bioelectron. , vol. 68, pp. 577-585, 2015.

[24] I. Giaever and C. R. Keese, "Micromotion of mammalian cells measured electrically," Proc. Natl. Acad. Sci. , vol. 88, no. 17, pp. 7896-7900, 1991.

[25] L. Ghenim et al. , "Monitoring impedance changes associated with motility and mitosis of a single cell," Lab Chip, vol. 10, no. 19, pp. 2546-2550, 2010.

[26] C. Xiao and J. H. T. Luong, "On-line monitoring of cell growth and cytotoxicity using electric cellsubstrate impedance sensing (ECIS)," Biotechnol. Prog., vol. 19, no. 3, pp. 1000-1005, 2003.

[27] L. Vistejnova et al. , "The comparison of impedance-based method of cell proliferation monitoring with commonly used metabolic-based techniques," Neuroendocrinol. Lett. , vol. 30, no. 1, p. 121, 2009.

[28] M. Abdolahad, M. Taghinejad, H. Taghinejad, M. Janmaleki, and S. Mohajerzadeh, "A vertically aligned carbon nanotube-based impedance sensing biosensor for rapid and high sensitive detection of cancer cells," Lab Chip , vol. 12, no. 6, pp. 1183-1190, 2012.

[29] S. K. Arya, K. C. Lee, and A. R. A. Rahman, "Breast tumor cell detection at single cell resolution using an electrochemical impedance technique," Lab Chip , vol. 12, no. 13, pp. 2362-2368, 2012.

[30] J.-L. Hong, K.-C. Lan, and L.-S. Jang, "Electrical characteristics analysis of various cancer cells using a microfluidic device based on single-cell impedance measurement," Sensors Actuators B Chem., vol. 173, pp. 927-934, 2012.

[31] J. Yun, Y.-T. Hong, K.-H. Hong, and J.-H. Lee, "Ex vivo identification of thyroid cancer tissue using electrical impedance spectroscopy on a needle," Sensors Actuators B Chem., vol. 261, pp. 537-544, 2018.

[32] H.-G. Jahnke et al. , "Impedance spectroscopy - an outstanding method for label-free and real-time discrimination between brain and tumor tissue in vivo," Biosens. Bioelectron., vol. 46, pp. 8-14, 2013.

[33] A. Han, L. Yang, and A. B. Frazier, "Quantification of the heterogeneity in breast cancer cell lines using whole-cell impedance spectroscopy," Clin. cancer Res. , vol. 13, no. 1, pp. 139-143, 2007.

[34] L. Yang, L. R. Arias, T. S. Lane, M. D. Yancey, and J. Mamouni, "Real-time electrical impedance-based measurement to distinguish oral cancer cells and non-cancer oral epithelial cells," Anal. Bioanal. Chem. , vol. 399, no. 5, pp. 1823-1833, 2011.

[35] M. Abdolahad, H. Shashaani, M. Janmaleki, and S. Mohajerzadeh, "Silicon nanograss based impedance biosensor for label free detection of rare metastatic cells among primary cancerous colon cells, suitable for more accurate cancer staging," Biosens. Bioelectron. , vol. 59, pp. 151-159, 2014.

[36] H. Guohua, L. Hongyang, J. Zhiming, Z. Danhua, and W. Haifang, "Study of small-cell lung cancer cell-based sensor and its applications in chemotherapy effects rapid evaluation for anticancer drugs," Biosens. Bioelectron. , vol. 97, pp. 184-195, 2017. 
[37] K. F. Lei, T.-K. Liu, and N.-M. Tsang, "Towards a high throughput impedimetric screening of chemosensitivity of cancer cells suspended in hydrogel and cultured in a paper substrate," Biosens. Bioelectron., vol. 100, pp. 355-360, 2018.

[38] C.-M. Lo, C. R. Keese, and I. Giaever, "Monitoring motion of confluent cells in tissue culture," Exp. Cell Res. , vol. 204, no. 1, pp. 102-109, 1993.

[39] M. Ashoorirad, A. Fallah, and M. Saviz, "Measuring and assessment of impedance spectrum of collagen thin films in the presence of deionized water," J. Mol. Liq. , vol. 320, p. 114488, 2020.

[40] A. L. Plant, K. Bhadriraju, T. A. Spurlin, and J. T. Elliott, "Cell response to matrix mechanics: focus on collagen," Biochim. Biophys. Acta (BBA)-Molecular Cell Res., vol. 1793, no. 5, pp. 893-902, 2009.

[41] A. L. Plant, J. T. Elliott, A. Tona, D. McDaniel, and K. J. Langenbach, "Tools for quantitative and validated measurements of cells," in High Content Screening , Springer, 2007, pp. 95-107.

[42] R. Ala-aho and V.-M. Kahari, "Collagenases in cancer," Biochimie, vol. 87, no. 3-4, pp. 273-286, 2005.

[43] K. Juurikka, G. S. Butler, T. Salo, P. Nyberg, and P. Astrom, "The Role of MMP8 in Cancer: A Systematic Review," Int. J. Mol. Sci., vol. 20, no. 18, p. 4506, 2019.

[44] C.-L. Hsiao et al. , "The association of matrix metalloproteinase-8 promoter genotypes in breast cancer," Anticancer Res. , vol. 38, no. 4, pp. 2181-2185, 2018. 